\title{
Papel de la detección regular de sustancias en orina en pacientes en valoración pre-trasplante hepático por hepatopatía alcohólica
} Role of Alcohol and Drug Detection by Regular Urine Sample
Testing in pre-transplant evaluation for Alcohol Liver Disease

\author{
Hugo López-Pelayo ${ }^{* * * *(*)}$, Jose Altamirano******(*), Eva López ${ }^{* * * * *}$, Pablo Barrio ${ }^{* * * * *}$, \\ Ana López ${ }^{*, * * *}$, Antoni GuAL ${ }^{*, * * * * *+}$, AnNa Lligoña ${ }^{*, * * *+}$. \\ * Grup Recerca Addicions Clínic (GRAC-GRE). Departamento de Psiquiatría, Instituto Clínico de Neurociencia. Hospital \\ Clínic i Universitari de Barcelona. Universitat de Barcelona. Villarroel, 170, 08036, Barcelona, España. ** Institut d'In- \\ vestigacions Biomèdiques August Pi i Sunyer (IDIBAPS), Rosselló, 149, 08036, Barcelona, España. *** Red de Trastornos \\ Adictivos. RETICS. C/ Sinesio Delgado, 4, 28029 Madrid, España. **** Instituto de Investigación Vall d'Hebron (VHIR), \\ Medicina Interna - Unidad de Transplante Hepático, Universidad Hospital Vall d'Hebron, Passeig de la Vall d'Hebron, 119, \\ 08035 Barcelona, España. ***** Unidad de Transplante Hepático. Hospital Clínic i Universitari de Barcelona. Universitat de \\ Barcelona. Villarroel, 170, 08036, Barcelona, España. (*) Primera autoría conjunta. ${ }^{\ddagger}$ Última autoría conjunta.
}

\section{Resumen}

La enfermedad hepática alcohólica (EHA) es una de las causas más frecuentes de trasplante hepático en enfermedad hepática terminal. No hay evidencia del impacto de la detección regular de sustancias en orina (DRSO) sobre la supervivencia de los pacientes con EHA. Los objetivos de este estudio fueron comparar la sensibilidad de la DRSO, evaluar su impacto en la supervivencia y en el trasplante hepático, y evaluar el impacto de la adherencia a la DRSO. Realizamos un estudio retrospectivo $(\mathrm{N}=84)$ con candidatos para trasplante hepático por EHA. Registramos las variables demográficas, bioquímicas y clínicas al inicio del estudio. Evaluamos la adherencia a la DRSO durante el seguimiento. Calculamos la sensibilidad tanto de la DRSO como de las declaraciones de los pacientes para todas las sustancias. Realizamos análisis multivariables (regresión logística) y de supervivencia para explorar los factores asociados y el impacto de la adherencia a la DRSO, y de los resultados positivos en la DRSO sobre la supervivencia. La DRSO tuvo una alta sensibilidad para identificar bebedores activos $(76,9 \%)$, fumadores $(78,9 \%)$ y consumidores de cannabis $(83,3 \%)$. La alta adherencia a la DRSO tuvo una asociación inversa con la mortalidad durante el seguimiento. La presencia de trastornos de la personalidad tuvo un impacto negativo (RM ,29, IC 95\%, 08-,97) sobre la adherencia a la DRSO. Tanto la DRSO como las declaraciones deben llevarse a cabo en este perfil de pacientes. Los profesionales que participan en programas de trasplante hepático deben promover el cumplimiento de la DRSO, principalmente en pacientes con trastornos de la personalidad. Palabras clave: Alcohol; Trasplante hepático; Enfermedad hepática terminal; Adherencia; Dependencia del alcohol; Dependencia de sustancias.

\begin{abstract}
Alcohol Liver Disease (ALD) is one of the most prevalent conditions leading to liver transplantation for end-stage liver disease. There is lacking evidence of regular urine screening testing (RUST) impact on survival or liver transplantation of ALD patients. The aims of this study were to compare the sensitivity of RUST, to assess its impact on survival and liver transplantation, and to evaluate factors associated with adherence to RUST. We performed a singlecentered retrospective study $(\mathrm{N}=84)$ with ALD candidates for liver transplantation. Demographic, biochemical and clinical variables were recorded at baseline. Adherence to RUST was evaluated during followup. The sensitivity of both RUST and self-reports were calculated for all drugs. Multivariable logistic and survival regression analyses were performed to explore associated factors and the impact of adherence to RUST, and positive results on survival. RUST had high sensitivity for identifying active drinkers $(76.9 \%)$, smokers $(78.9 \%)$ and cannabis users $(83.3 \%)$. High adherence to RUST was inversely associated with mortality during follow-up. Presence of personality disorders negatively impacted (OR 0.29, CI 95\% 0.08-0.97) adherence to RUST. Both RUST and self-reports should be carried out in this setting. Professionals involved in liver transplantation programs must promote adherence to RUST, primarily in patients with personality disorders.

Keywords: Alcohol; Liver transplantation; Alcohol liver disease; Adherence; Alcohol dependence; Drug dependence.
\end{abstract}

Recibido: Febrero 2018; Aceptado: Abril 2018.

Enviar correspondencia a:

Hugo López-Pelayo. Hospital Clínic i Universitari de Barcelona. Villarroel 170, escalera 9 planta 6, 08036, Barcelona, España. Tel: +34 932271719. Fax: +34932275548. Email: hlopez@clinic.cat 


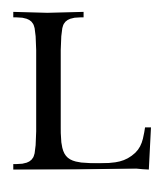

a enfermedad hepática alcohólica (EHA) es una de las causas principales de morbilidad y mortalidad a nivel mundial (Rehm, Gmel, Sierra y Gual, 2018) y una de las causas principales de enfermedad hepática terminal (EHT) y muerte entre adultos con trastorno por uso de alcohol (Mathurin y Bataller, 2015). El trasplante hepático (TH) es un tratamiento bien establecido para la EHT como resultado de la EHA (Dawwas, Gimson, Lewsey, Copley y van der Meulen, 2007). La supervivencia tras un trasplante hepático en pacientes con EHA es similar a la de los pacientes con otras etiologías de EHA (Burra et al., 2010).

La prevalencia de metabolitos de drogas o alcohol en pacientes con EHA que son candidatos para un trasplante hepático es alta (Carbonneau et al., 2010; Erim et al., 2007; Webzell et al., 2011). La mayoría de los programas de trasplante hepático requieren una abstinencia de 6 meses para el alcohol y de 12 meses para las otras drogas para incluir a los pacientes en la lista de espera (Beresford y Everson, 2000; Lligoña, Freixa, Bataller, Monràs y Rimola, 2009). No obstante, se consideran algunas excepciones (p.ej., cannabis o tabaco) (Lligoña et al., 2009). Aun así, el consenso internacional es inexistente.

En nuestro programa, la evaluación de los pacientes candidatos a un trasplante hepático incluye entrevistas psicológicas y psiquiátricas con el fin de identificar la presencia de trastornos psiquiátricos que puedan impedir el trasplante. Dichas entrevistas también valoran el uso del alcohol y otras drogas como parte de los protocolos estándar. Se recomienda la detección de alcohol y otras drogas mediante la detección regular de sustancias en orina (DRSO) al menos semanalmente en el seguimiento de estos pacientes (Lligoña et al., 2009). No obstante, la evidencia es mixta sobre la DRSO para monitorizar pacientes con dependencia del alcohol (p.ej., semivida corta) (Barrio et al., 2016; Niemelä, 2016) y es limitada sobre candidatos a un trasplante hepático por EHA (Allen, Wurst, Thon, y Litten, 2013; Carbonneau et al., 2010; Piano et al., 2014; Staufer y Yegles, 2016; Webzell et al., 2011). Es más, la mayoría de estos estudios se realizó siguiendo protocolos de investigación, en los cuales la adherencia se podía haber sobrestimado por el efecto Hawthorne y, por tanto, también la validez de la DRSO. Además, los autoinformes se consideran una estrategia válida para valorar el uso de alcohol y drogas en muchas condiciones, incluso en ensayos clínicos (CDER, 2015; EMA, 2010). Es más, los autoinformes sobre el uso de alcohol y drogas es una estrategia coste-efectiva. No obstante, los candidatos a un trasplante hepático tienen la consideración de ser una población especial porque pueden subestimar su consumo de alcohol si piensan que su ingesta actual pueda retrasar o contraindicar el trasplante (Allen et al., 2013).

Por otro lado, la adherencia al tratamiento predice buenos resultados en varias condiciones incluyendo los trastor- nos por uso de alcohol (Oslin, Pettinati y Volpicelli, 2002) y EHA (Rustad, Stern, Prabhakar y Musselman, 2015; Telles-Correia, Barbosa, Mega y Monteiro, 2009). De todas maneras, no hay suficientes estudios sobre el impacto de la adherencia a la DRSO o sobre sus resultados positivos en el pronóstico para pacientes con EHA.

Los objetivos de este estudio, centrado en pacientes valorados para un trasplante hepático, fueron: 1) comparar la sensibilidad de los autoinformes y la DRSO en la detección del uso de alcohol/drogas; 2) valorar el impacto de la adherencia a la DRSO y sus resultados positivos sobre el trasplante hepático y la supervivencia; y 3 ) explorar los factores basales asociados con una adherencia alta a la DRSO.

\section{Materiales y método}

\section{Diseño del estudio}

Estudio observacional retrospectivo (post-hoc) en un centro único.

\section{Participantes}

Reclutamos a todos los pacientes con EHA mayores de 17 años, evaluados de forma consecutiva en la etapa pre-trasplante (aceptados o no en la lista de espera para un trasplante hepático) del Servicio Ambulatorio de Trasplante Hepático entre enero del 2008 y enero del 2014.

Criterios de exclusión: pacientes que no realizaron la DRSO en nuestra unidad de adicciones, sino en otros lugares, debido a restricciones geográficas.

\section{Entorno}

El protocolo de evaluación actual para trasplante hepático en pacientes con EHA en el Hospital Clínic Barcelona requiere una valoración exhaustiva por un psiquiatra y un psicólogo, y la DRSO para las sustancias que con mayor frecuencia son objeto de abuso (alcohol, benzodiazepinas, nicotina, cocaína, opiáceos y cannabis) al menos una vez por semana (Lligoña et al., 2009). Se determinó la frecuencia de la DRSO según criterios clínicos del psiquiatra del paciente, que tuvo en cuenta las drogas primarias y secundarias usadas y la capacidad personal de los pacientes para realizar la DRSO (restricciones físicas, geográficas, laborales o familiares). Lo habitual es que el psiquiatra prescribiese la DRSO dos veces por semana, según la práctica clínica convencional, las dificultades para visitar la consulta y la disponibilidad de recursos en nuestras clínicas ambulatorias. Dos veces por semana es suficiente para identificar la recaída a las drogas por la semivida larga de la mayoría de las drogas y el consumo habitual de alcohol, pero es insuficiente para detectar el uso de alcohol ocasional. No obstante, así era la práctica real en nuestro entorno entre el 2008-2014, y antes de la generalización de étil glucurónido en muestra de orina, que se comenzó a utilizar en nuestro hospital en octubre 2016. 
Comenzamos el reclutamiento en enero 2008 cuando el Comité de Ética de HCB aprobó el protocolo actual para el trasplante hepático en pacientes con EHA. Nuestros datos hacen referencia a los seis años iniciales desde la implementación del protocolo. El seguimiento comenzó con la primera cita con el psiquiatra y finalizó con el fallecimiento del paciente o en fecha de octubre 2015.

La recolección de datos se realizó de forma prospectiva durante la valoración para el trasplante hepático, exceptuando el MELD (Modelo para Enfermedad Hepática Terminal), la adherencia a la DRSO y el seguimiento, la supervivencia y la realización del trasplante hepático, cuyos datos fueron recopilados en el último trimestre del 2015.

\section{Variables}

Registramos los siguientes datos, de forma sistemática y prospectiva, durante la entrevista psiquiátrica:

1) Datos demográficos: edad (en la fecha de la primera muestra de orina) y sexo,

2) Datos psiquiátricos y clínicos:

- Historia actual y pasada de trastorno psiquiátricos según criterios del DSM IV-TR basados en el diagnóstico clínico del psiquiatra responsable: incluyendo Trastornos de Estado de Ánimo (depresión, distimia, trastorno bipolar), Trastornos Psicóticos (esquizofrenia, trastorno esquizoafectivo, otros trastornos relacionados) y Trastornos de Ansiedad (trastorno obsesivo-compulsivo, trastorno de pánico, trastorno de ansiedad generalizada, trastorno por estrés postraumático, fobia). Los Trastornos de Personalidad fueron agrupados en tres categorías, según la clasificación del DSM IV-TR: A (trastornos de la personalidad paranoide, esquizoide y esquizotípico), B (trastorno límite de la personalidad, trastorno narcisista de personalidad, trastorno histriónico de la personalidad, y trastorno de personalidad antisocial) y C (trastornos de la personalidad por evitación, por dependencia y obsesivo-compulsivo).

- Patrón de uso de alcohol actual y a lo largo de la vida: usando el cuestionario sobre frecuencia y cantidades llamado ISCA (Interrogatorio Sistematizado de Consumos Alcohólicos) (Gual, Contel, Segura, Ribas y Colom, 2001). Las sensibilidades del ISCA fueron $70-81 \%$ para hombres y $46-100 \%$ para mujeres. Las especificidades del ISCA fueron $82-99 \%$ y $97-100 \%$, respectivamente.

- Patrón de uso de drogas actual y a lo largo de la vida. - La puntuación del High-Risk Alcohol Relapse (HRAR): HRAR es una escala de 3 ítems que valora el consumo total de alcohol diario, los años de consumo de riesgo de alcohol y los tratamientos anteriores por trastorno por uso de alcohol. Este sistema asigna una puntuación entre 0-2 para cada ítem evaluado (puntuación total máxima de 6), estratificando a los pacientes en 2 categorías de riesgo de recaída en el consumo de alcohol (alto riesgo $(<4)$ o bajo riesgo $(\geq 4))$. Una puntuación en HRAR superior a 3 es un factor de riesgo para la recaída al alcoholismo tras el trasplante hepático (RM, 10,7; IC 95\%, 3,8-30,0) (De

Gottardi et al., 2007; DiMartini et al., 2000).

Al final del estudio, usamos los registros clínicos para hacer una recopilación retrospectiva de los datos que siguen:

1) Cuantía de muestras de orina programadas.

2) Cuantía de muestras de orina completadas.

3) Seguimiento en la Unidad de Adicciones y/o Hepatología.

4) Realización del trasplante hepático.

5) Variable de supervivencia (Fallecido/Vivo): Para los fines de este estudio, la variable combinada de vivo en el último seguimiento o trasplante hepático fue la principal usada para el análisis de supervivencia.

6) Adherencia a la DRSO: Calculamos la adherencia de la siguiente manera: (número de muestras de orina programadas/Cuantía de muestras de orina completadas) x 100. Estratificamos la adherencia en 3 categorías: adherencia baja $(<25 \%)$, adherencia intermedia $(25-75 \%)$ y adherencia alta $(\geq 75 \%)$. Consideramos dos categorías para el análisis inferencial: adherencia alta $(\geq 75 \%)$ vs. adherencia no-alta $(<75 \%)$.

7) Model for End-stage Liver Disease (MELD) en el momento del primer análisis de orina (Malinchoc et al., 2000).

Analizamos los metabolitos que siguen, usando los puntos de corte fijados por el laboratorio del Hospital Clínic y el periodo de detección:

1) Alcohol: etanol (punto de corte: $100 \mathrm{ng} / \mathrm{mL}$; periodo de detección $\leq 12 \mathrm{~h}$ )

2) Tabaco: cotinina (punto de corte: $100 \mathrm{ng} / \mathrm{mL}$; periodo de detección: 3 días)

3) Opioides: morfina (punto de corte: $300 \mathrm{ng} / \mathrm{mL}$; periodo de detección: 3 días)

4) Cocaína: benzoilecgonina (punto de corte: $300 \mathrm{ng} /$ mL; periodo de detección: 3 días)

5) Benzodiazepinas: diazepam (punto de corte: $200 \mathrm{ng} /$ $\mathrm{mL}$; periodo de detección: 10 días-5 semanas)

6) Cannabis: 11-nor-d9-THC (punto de corte: $50 \mathrm{ng} / \mathrm{mL}$; periodo de detección: 5 días-5 semanas)

\section{Análisis estadístico}

Realizamos un análisis descriptivo de la muestra. Describimos las variables continuas con la media y la desviación estándar. Las variables categóricas las describimos por número y porcentajes. Implementamos comparaciones entre grupos usando la prueba $t$ de Student, el análisis de varianza (ANOVA) o la prueba $U$ de Mann-Whitney, según la distribución de las variables. Analizamos las diferencias entre las variables categóricas usando el test de chi cuadrado o el test exacto de Fisher, cuando procedía. Los valores $\mathrm{p}<, 05$ fueron considerados significativos. 
Calculamos la sensibilidad y el área bajo la curva de forma individual para los autoinformes y la DRSO usando el conjunto de ambos métodos (autoinformes y DRSO) como el estándar de oro (objetivo 1). Elegimos este estándar de oro - dadas nuestras experiencias previas con protocolos de investigación en los cuales ambos resultados por separado mostraron menor sensibilidad- porque el resultado combinado identifica el número más alto de usuarios activos. Con el fin de investigar las variables con valor pronóstico para la variable combinada (vivo/trasplante hepático) durante el seguimiento de los pacientes (objetivo 2), adaptamos los análisis de regresión unifactorial y multivariable de Cox, incluyendo los variables de la evaluación inicial y durante el seguimiento. Consideramos los resultados del análisis de regresión multivariable de Cox (tasa de riesgo -TR-) como el resultado principal. Con el fin de evaluar la influencia de la adherencia a la DRSO en la supervivencia de los pacientes durante el seguimiento (objetivo 2), realizamos un análisis comparativo de riesgo con el método Kaplan-Meier (prueba logrank). Para estudiar las variables asociadas con una adherencia alta a la DRSO (objetivo 3), aquellos variables con una $\mathrm{p}<, 10$ y aquellos que se consideraron de relevancia clínica en el análisis unifactorial, se incluyeron en un backward stepwise elimination variable selection procedure (procedimiento de regresión logística de eliminación hacia atrás). Al considerar los tests exploratorios, los valores p para las pruebas monofactoriales no fueron corregidos para pruebas múltiples. Usamos el paquete estadístico SPSS (SPSS Inc., versión 15.0, Chicago, IL) para todos los análisis.

\section{Cuestiones éticas}

El Comité de Ética del Hospital Clínic de Barcelona aprobó este estudio (HCB/2015/0845), según normas de la Declaración de Helsinki y legislación española. Dado el diseño retrospectivo, no fue necesario el consentimiento informado; usamos únicamente información clínica rutinaria y después de haber garantizado a los participantes su anonimidad absoluta.

\section{Resultados}

\section{Características de los pacientes}

La muestra definitiva estaba compuesta por 84 pacientes. El 88,1\% eran hombres y con una edad media de 53,7 (DE 6,2) años. Al llegar al final del estudio, el 67,9\% ( $\mathrm{n}=$ 57) seguía con vida y el $34,5 \%(n=29)$ había recibido un trasplante hepático. La media del MELD en la fecha de la primera muestra de orina fue 14,2 (DE 5,6). La media de seguimiento, en general, fue 15,9 (DE 11,4) meses. La tabla 1 describe ampliamente las características clínicas.

El 29,8\% de los pacientes tenía un trastorno mental, con un trastorno de la personalidad del grupo B como el diagnóstico más frecuente $(\mathrm{n}=9 ; 10,7 \%)$. Otros diagnósticos incluyeron trastorno de depresión $(n=6 ; 7,1 \%)$, trastorno
Tabla 1. Características sociodemográficas y clínicas de los pacientes con Enfermedad Hepática Alcohólica evaluados en la valoración previa al trasplante hepático.

\begin{tabular}{|c|c|c|}
\hline & & n (\%) \\
\hline Sexo (hombre) & & $74(88,1)$ \\
\hline Edad (media, DE) & & $53,7(6,2)$ \\
\hline MELD basal (media, DE) & & $14,2(5,6)$ \\
\hline Trastorno mental (actual o anterior) & & $25(29,8)$ \\
\hline \multirow[t]{3}{*}{$\mathrm{UBE}^{\mathrm{a}}$ /día } & $\iota=9$ & $40(47,6)$ \\
\hline & $9-17$ & $36(42,9)$ \\
\hline & $>17$ & $8(9,5)$ \\
\hline \multirow[t]{3}{*}{ Años de consumo abusivo } & $\ll=11$ & $26(31)$ \\
\hline & $11-25$ & $40(47,6)$ \\
\hline & $>25$ & $18(21,4)$ \\
\hline \multirow{3}{*}{$\begin{array}{l}\text { Tratamiento anterior para trastorno } \\
\text { por uso de alcohol }\end{array}$} & 0 & $65(77,4)$ \\
\hline & 1 & $13(15,5)$ \\
\hline & $>1$ & $6(7,1)$ \\
\hline \multirow[t]{2}{*}{$\mathrm{HRAR}^{\mathrm{b}}$} & Riesgo bajo ( $(4)$ & $74(88,1)$ \\
\hline & Riesgo alto $(=>4)$ & $10(11,9)$ \\
\hline Fallecimiento & & $27(32,1)$ \\
\hline Seguimiento en la Unidad de Adicciones & & $13(15,5)$ \\
\hline Seguimiento en la Unidad de Hepatología & & $56(66,7)$ \\
\hline Uso de alcohol a lo largo de la vida & & $84(100)$ \\
\hline $\begin{array}{l}\text { Uso de benzodiazepinas a lo largo } \\
\text { de la vida }\end{array}$ & & $10(11,9)$ \\
\hline Uso de cannabis a lo largo de la vida & & $28(33,3)$ \\
\hline Uso de cocaína a lo largo de la vida & & $12(14,3)$ \\
\hline Uso de tabaco a lo largo de la vida & & $39(46,4)$ \\
\hline Uso de opiáceos a lo largo de la vida & & $11(13,1)$ \\
\hline
\end{tabular}

Nota. aUBE: Unidad de Bebida Estándar (10 gramos de alcohol puro); ${ }^{\mathrm{b} H R A R:}$ Alto Riesgo de Recaída al Alcoholismo.

de ansiedad $(\mathrm{n}=4 ; 4,8 \%)$, trastorno de personalidad del grupo A $(n=3 ; 3,6 \%)$ y trastorno de personalidad del grupo $\mathrm{C}(\mathrm{n}=3 ; 3,6 \%)$.

\section{Objetivo 1: comparación de la sensibilidad de los autoinformes y la DRSO para la Detección de Uso de Alcohol y Drogas (variable combinada de referencia)}

Tabla 3 muestra la sensibilidad y el área bajo la curva usando muestras de orina + autoinformes como estándar de oro. Para el caso de alcohol, cannabis y tabaco, la sensibilidad es mejor para la DRSO que para los autoinformes, y es peor para benzodiazepinas y cocaína.

\section{Objetivo 2. Impacto de la DRSO (adherencia y resultados) respecto de Trasplante Hepático y Mortalidad}

Adherencia/al menos un positivo en la DRSO y Trasplante Hepático. Estudiamos las variables con una asociación potencial 
Tabla 2. Adherencia y resultados positivos en muestra de orina en la valoración previa al trasplante hepático en pacientes con Enfermedad Hepática Alcohólica.

\begin{tabular}{|c|c|c|c|c|}
\hline & & $\begin{array}{r}\text { Muestra total } \\
(\mathrm{N}=84) \\
\mathrm{n}(\%)\end{array}$ & $\begin{array}{r}\text { Con trastorno } \\
\text { psiquiátrico } \\
(\mathrm{n}=25) \\
\mathrm{n}(\%)\end{array}$ & $\begin{array}{r}\text { Sin trastorno } \\
\text { psiquiátrico } \\
(\mathrm{n}=59) \\
\mathrm{n}(\%)\end{array}$ \\
\hline \multirow[t]{3}{*}{ Adherencia a la DRSO } & Baja $(\iota=25 \%)$ & $5(6)$ & $3(12)$ & $2(3,4)$ \\
\hline & Intermedia (26-75\%) & $27(32,1)$ & $10(40)$ & $17(28,8)$ \\
\hline & Alta ( $(=75 \%)$ & $52(61,9)$ & $12(48)$ & $40(67,8)$ \\
\hline \multirow[t]{2}{*}{ Adherencia media de DRSO (media, DE) } & & $35(30,1)$ & $37,5(35,8)$ & $33,9(27,6)$ \\
\hline & & & & $\mathrm{n}(\%)$ \\
\hline \multicolumn{3}{|c|}{ Número de pacientes con al menos una muestra de orina positiva para alcohol } & & $10(11,9)$ \\
\hline \multicolumn{3}{|c|}{ Número de pacientes con al menos una muestra de orina positiva para benzodiazepinas } & & $8(9,5)$ \\
\hline \multicolumn{3}{|c|}{ Número de pacientes con al menos una muestra de orina positiva para cannabis } & & $15(17,9)$ \\
\hline \multicolumn{3}{|c|}{ Número de pacientes con al menos una muestra de orina positiva para cotinina } & & $30(35,7)$ \\
\hline \multicolumn{3}{|c|}{ Número de pacientes que autoinformó el uso actual de alcohol } & & $6(7,1)$ \\
\hline \multicolumn{3}{|c|}{ Número de pacientes que autoinformó el uso actual de benzodiazepinas } & & $12(14,3)$ \\
\hline \multicolumn{3}{|c|}{ Número de pacientes que autoinformó el uso actual de cannabis } & & $12(14,3)$ \\
\hline \multicolumn{3}{|c|}{ Número de pacientes que autoinformó el uso actual de cocaína } & & $2(2,4)$ \\
\hline \multicolumn{3}{|c|}{ Número de pacientes que autoinformó el uso actual de cotinina } & & $23(27,4)$ \\
\hline
\end{tabular}

Nota. ${ }^{a}$ No hubo casos positivos para opiáceos o cocaína en la DRSO, o para opiáceos en los autoinformes.

Tabla 3. Características psicométricas de los autoinformes o de la DRSO (variable combinada de referencia DRSO + autoinforme) en pacientes con Enfermedad Hepática Alcohólica evaluados en la valoración previa al trasplante hepático.

\begin{tabular}{|c|c|c|c|}
\hline & Autoinforme & $\begin{array}{l}\text { Detección Regular } \\
\text { de Sustancias en Orina }\end{array}$ & $\begin{array}{l}\text { Diferencias (Detección Regular de } \\
\text { Sustancias en Orina - autoinformado) }\end{array}$ \\
\hline \multicolumn{4}{|c|}{ Usuarios de alcohol $(n=13,15,5 \%)^{c}$} \\
\hline Sensibilidad (\%) & 46,2 & 76.9 & 30.7 \\
\hline${ }^{\mathrm{a}} \mathrm{ABC}$ & ,73 (IC 95\%, 549-,913) & ,89(IC 95\%,00-1,00) & 0.16 \\
\hline \multicolumn{4}{|c|}{ Usuarios de benzodiazepinas $(n=17,20,2 \%)^{c}$} \\
\hline Sensibilidad (\%) & 70,6 & 47.1 & -23.5 \\
\hline${ }^{\mathrm{a} A B C}$ & ,85 (IC 95\%,72-,99) & ,74 (IC 95\%, 58-,90) & -0.11 \\
\hline \multicolumn{4}{|c|}{ Usuarios de cannabis $(n=18,21,7 \%)^{c}$} \\
\hline Sensibilidad (\%) & 66,7 & 83,3 & 16,6 \\
\hline$A B C$ & ,83 (IC 95\%,70-1,00) & ,92 (IC 95\%,00-1,00) &, 09 \\
\hline \multicolumn{4}{|c|}{ Usuarios de cocaína $(n=2,2,4 \%)^{c}$} \\
\hline Sensibilidad (\%) & 100 & 0 & -100 \\
\hline${ }^{\mathrm{a} A B C}$ & 1,00 (IC 95\% 1,00-1,00) & ,5 (IC 95\%,09-,91) &,- 5 \\
\hline \multicolumn{4}{|c|}{ Usuarios de tabaco $(n=38,45,2 \%)^{c}$} \\
\hline Sensibilidad (\%) & 60,5 & 78,9 & 18,9 \\
\hline${ }^{\mathrm{a} A B C}$ & ,80 (IC 95\%,70-,91) & ,90 (IC 95\%,82-,97) & ,10 \\
\hline
\end{tabular}

Nota. ${ }^{\mathrm{A} A B C}$ : Área Bajo la Curva; 'C: Intervalo de Confianza. 'Según el estándar de oro combinado (DRSO + autoinformes). 
con el trasplante hepático $(n=29)$ en los pacientes incluidos. La adherencia (adherencia alta $\mathrm{n}=19,37,3 \%$ versus adherencia no-alta $\mathrm{n}=10,30,3 \% ; \mathrm{p}=, 531)$ no estaba asociada con trasplante hepático. Tampoco estaban asociados con el resultado del trasplante hepático al menos un resultado positivo para alcohol (30\% versus $35,1 \% ; \mathrm{p}=, 749)$, al menos un resultado positivo para nicotina $(23,3 \%$ versus $40,7 \% ; \mathrm{p}=, 108)$ o al menos un resultado positivo para otras sustancias $(38,1 \%$ versus $33,3 \% ; p=, 89)$. La tabla 4 muestra los criterios de exclusión para trasplante hepático. Adherencia/Al menos un positivo en la DRSO y Mortalidad. Por último, estudiamos los variables asociados con la mortalidad durante el seguimiento de los pacientes incluidos. La adherencia media fue similar entre los que habían recibido un trasplante hepático o que estaban vivos al finalizar el estudio y los pacientes que habían fallecido (74,5\% versus $64,6 \% ; \mathrm{p}=, 08)$ pero era menos probable que se clasificase como adherencia alta si falleció (71,9\% versus $37 \%$, p $=, 02)$. Excluyendo a los pacientes que recibieron un trasplante hepático (población diana $=55$ ), al finalizar el estudio seguían vivos el 26,1\% $(n=6)$ de los pacientes con adherencia baja y el $73,3 \%(\mathrm{n}=22)$ de los pacientes con adherencia alta $(\mathrm{p}=, 003)$. Los análisis de regresión de Cox identificaron la adherencia alta a la DRSO y una asociación positiva entre puntuación en HRAR y mortalidad durante el seguimiento. Con el fin de profundizar en el análisis del valor independiente de los variables predictores de mortalidad, las variables estadísticamente significantes y clínicamente relevantes se incluyeron en un modelo multivariable final. Encontramos que una adherencia baja a la DRSO (TR,44; $p=, 04$ ), puntuación HRAR > 3 puntos (TR 2,95; $p=, 02$ ) y puntuación MELD (TR 1,$08 ; p=, 03$ ) tuvieron una asociación independiente con mortalidad durante el seguimiento. De los 51 pacientes con adherencia alta a la DRSO, 22 (43\%) seguían vivos (sin trasplante hepático) hasta el último seguimiento (tiempo medio de seguimiento 15 [DE 10] meses) y $19(37,3 \%)$ pacientes habían recibido un trasplante hepático (tiempo medio hasta TH: 11 [DE 5] meses). Los pacientes con una adherencia baja a la DRSO mostraron una mortalidad más alta, comparado con los pacientes con una adherencia alta $(17 / 33$ [51,5\%] vs. $10 / 51[19,6 \%] ; p=, 01)$. Por último, una adherencia alta a la DRSO tuvo un impacto positivo en la supervivencia general en el último seguimiento (Figura 1). La presencia

Tabla 4. Criterios de exclusión para trasplante hepático.

\begin{tabular}{lr}
\hline Motivo & $\mathbf{n}(\%)$ \\
\hline Bebedores activos & $15(27,3 \%)$ \\
No-adherencia a la DRSO* & $11(20 \%)$ \\
Mejora de la enfermedad hepática & $9(16,4 \%)$ \\
Fallecimiento durante la valoración & $1(1,8 \%)$ \\
\hline
\end{tabular}

Nota. * Detección Regular de Sustancias en Orina.

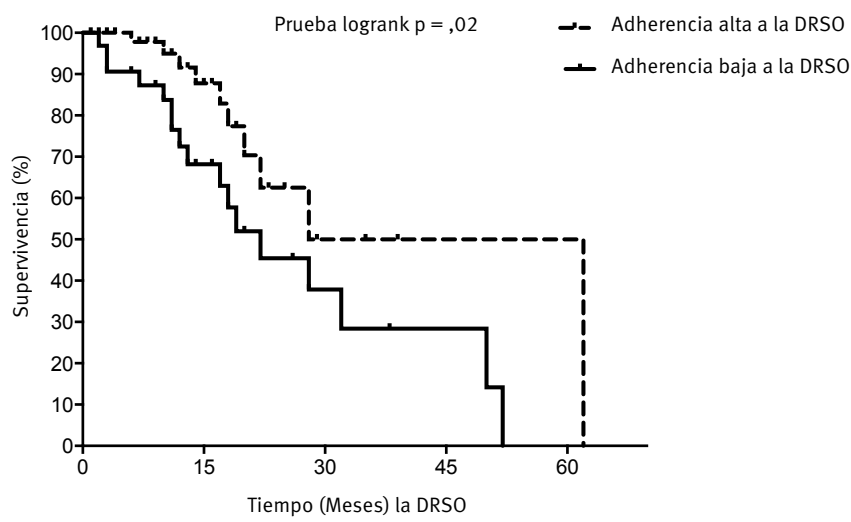

Figura 1. Supervivencia según adherencia a la DRSO durante seguimiento de pacientes con EHA.

Tabla 5. Análisis monofactoriales y multivariables de los factores asociados con la adherencia alta a la DRSO en la valoración previa al trasplante hepático en pacientes con Enfermedad Hepática Alcohólica durante el seguimiento desde su registro (primera cita con el psiquiatra).

\begin{tabular}{|c|c|c|c|c|c|c|}
\hline \multirow[b]{2}{*}{ Variable } & \multicolumn{3}{|c|}{ Análisis monofactorial } & \multicolumn{3}{|c|}{ Análisis multivariable } \\
\hline & RM & IC $95 \%$ & $\mathbf{p}$ & RM & IC $95 \%$ & $\mathbf{p}$ \\
\hline Edad (años) & 1,03 & $, 96-1,11$ &, 5 & & & \\
\hline Sexo (mujer) &, 38 & $, 10-1,47$ &, 2 & & & \\
\hline Comorbilidad psiquiátrica (s/n) & ,37 &, $14-, 98$ &, 04 & & & \\
\hline Trastorno de personalidad (s/n) &, 25 &, $08-, 82$ &, 02 &, 29 & ,08 -,97 &, 04 \\
\hline Otras drogas de abuso* $(\mathrm{s} / \mathrm{n})$ & ,68 & $, 28-1,65$ &, 4 & & & \\
\hline $\begin{array}{l}\text { Puntuación HRAR al ingreso del paciente } \\
\text { (puntos) }\end{array}$ &, 76 & $, 56-1,03$ & ,08 & ,81 & $, 59-1,11$ & ,2 \\
\hline Puntuación HRAR al ingreso ( $>3$ puntos) & ,38 & ,09-1,47 & ,16 & & & \\
\hline
\end{tabular}


de al menos un resultado positivo para alcohol, nicotina $u$ otras sustancias durante el seguimiento no estuvo asociada con la mortalidad (datos no mostrados).

\section{Objetivo 3: Factores basales que predicen la adherencia a la DRSO}

En general, la adherencia media fue $71,3 \%$ (DE 24,4). Durante el seguimiento, el 60,7\% (51 de los 84 pacientes) mostró una adherencia alta a la DRSO; en el caso de los pacientes que habían recibido un trasplante hepático, la adherencia alta estaba presente en el $66 \%$ de ellos.. No hallamos diferencias en la duración del seguimiento entre pacientes con una adherencia alta vs. adherencia baja a la DRSO (16,9 versus 15,3 meses, respectivamente; $p=$ ,52). En el análisis monofactorial, la presencia de trastorno mental, trastorno de personalidad y puntuaciones HRAR tenía una asociación negativa con adherencia alta a la DRSO (Tabla 5). Los pacientes con trastornos de la personalidad (TP) tenían menor adherencia a la DRSO que los pacientes sin esta condición ( $31 \%$ vs. $10 \% ; p=, 02)$. $\mathrm{Al}$ incluir estos variables en el análisis multivariable, solo la presencia de un trastorno de personalidad (RM ,29; $p$ $=, 04)$ mostró una asociación negativa e independiente con adherencia alta a la DRSO. Hemos de mencionar que la puntuación HRAR no alcanzó significación estadística cuando la ajustamos para otras covariables. Por último, no hallamos ninguna diferencia en la duración total del seguimiento en pacientes con o sin algún trastorno mental o con o sin un trastorno de la personalidad (15,7 vs. 16,1 meses; $p=, 8$ y 15,8 vs. 16,2 meses; $p=, 9$, respectivamente).

\section{Discusión}

La valoración de pacientes con EHA en proceso de un trasplante hepático supone un reto tanto para hepatólogos como para psiquiatras. Las valoraciones iniciales incluyen entrevistas psiquiátricas y psicológicas con el fin de identificar trastornos psiquiátricos, que podrían ser una causa potencial de exclusión de la lista de espera (Bunzel y Laederach-Hofmann, 2000; Martin, DiMartini, Feng, Brown y Fallon, 2014; Surman, Cosimi y DiMartini, 2009). Aunque la duración de la abstinencia es objeto de debate para pacientes con EHA (Di Martino, Sheppard y Vanlemmens, 2012) que son candidatos potenciales para un trasplante hepático, un periodo de abstinencia de $>6$ meses antes del trasplante hepático es uno de los criterios aplicados con mayor frecuencia en los centros de trasplante hepático y en las guías clínicas ("EASL Clinical Practice Guidelines: Liver transplantation," 2015; Lligoña et al., 2009; Martin et al., 2014). Esta "regla de los 6 meses" se ha propuesto como una necesidad para limitar la recaída en el consumo de alcohol de alto riesgo que podría poner en peligro la integridad del injerto (Allen et al., 2013). Sin duda, una proporción de los pacientes que reciben un trasplante hepático vuelve a beber que eventualmente resulta en la pérdida del injerto o en fallecimiento, debido al incumplimiento de los tratamientos inmunosupresores o por las lesiones hepáticas causadas directamente por el consumo de alcohol (Cuadrado, Fábrega, Casafont y Pons-Romero, 2005). Por tanto, la monitorización de la abstinencia y del cumplimiento de los protocolos por los pacientes en el entorno pre-trasplante es de importancia absoluta y un paso crítico en el proceso de valoración de pacientes con EHA para trasplante hepático. El uso de autoinformes es una estrategia válida y coste-efectiva para valorar el uso de alcohol y otras drogas en muchos escenarios clínicos (EMA, 2010). No obstante, en el proceso del trasplante hepático, los autoinformes han mostrado una precisión baja para identificar a los bebedores activos, dada la tasa alta de información incompleta (Allen et al., 2013).

Sensibilidad de autoinformes y la DRSO. En nuestra muestra, la DRSO mostró mejor sensibilidad para identificar a los bebedores activos y usuarios de drogas activos, con la excepción de benzodiazepinas y/o cocaína, pero no identificó a todos los usuarios activos. La detección de alcohol y benzodiazepinas en las muestras de orina fue en una proporción similar a otras muestras de candidatos a un trasplante hepático con EHA (Webzell et al., 2011). Por lo contrario, no pudimos identificar caso alguno de consumo de opiáceos cuando el uso de estas drogas fue informado por hasta el $20 \%$ de los pacientes con EHA candidatos a un trasplante hepático en otros países (p.ej., Reino Unido). Dichas diferencias pueden explicarse por la variabilidad en los patrones de consumo en cada país y también por diferencias técnicas para medir sustancias entre las diferentes muestras poblacionales (EMCDDA, 2015; Stewart, Koch, Burgess, Willner y Reuben, 2013; Wurst et al., 2003).

El uso de la DRSO para alcohol permite identificar casi $31 \%$ más casos que los autoinformes, pero no logró identificar a todos los usuarios activos $(23,1 \%$ de falsos negativos en la DRSO en nuestro estudio). Los autoinformes identificaron menos de uno de cada dos usuarios de alcohol actuales. Nuestros resultados son coherentes con otros estudios recientes sobre tratamiento del trastorno por uso de alcohol en candidatos a un trasplante hepático (Erim et al., 2016). Mediante la DRSO, pudimos detectar a casi ocho de cada diez usuarios de alcohol, aunque no pudimos identificar a todos los usuarios de alcohol. Por lo tanto, combinar autoinformes y la DRSO es la mejor estrategia, confirmando las recomendaciones de las guías clínicas ("EASL clinical practical guidelines: management of alcoholic liver disease”, 2012). Además, la información insuficiente es más alta en nuestra población que en otras poblaciones sensibles, como pacientes ingresados por trastornos psiquiátricos $(56 \%)$ o mujeres embarazadas $(0 \%)$ (de Beaurepaire et al., 2007; Horrigan, Piazza y Weinstein, 1996). La baja comunicación del uso de alcohol quiere decir que 
su consumo se percibe como una contraindicación para el trasplante hepático en un contexto de alta resistencia para reconocer su uso. Es más, nuestros análisis de laboratorio son menos sensibles que la étil glucurónido en muestra de orina (EGMO) que es posiblemente más potente para detectar cualquier consumo reciente de alcohol, tiene propiedades validadas excelentes en pacientes con enfermedad hepática, y una fuerte correlación con la cantidad de alcohol consumido (Nanau y Neuman, 2015; Wurst et al., 2015). Un estudio reciente de nuestro grupo muestra que la abstinencia estaba presente en el $95 \%$ de los pacientes según el etanol en orina, sin embargo, esta cifra descendía al 60\% con EGMO (Barrio et al., 2016b). Según un estudio reciente con candidatos a un $\mathrm{TH}$, el diagnóstico más preciso del consumo de alcohol se logró combinando la versión abreviada del Alcohol Use Disorder Identification Test (AUDIT-C) y EGMO (curva ROC ,98) (Piano et al., 2014).

La baja comunicación de uso de consumo de tabaco se explica por la actitud defensiva del paciente, en general, ante la valoración del uso de drogas durante la evaluación para un trasplante hepático (Allen et al., 2013). La baja comunicación de uso de consumo de cánnabis es similar al identificado en meta-análisis reciente (19 estudios) que informan una sensibilidad del ,60 para autoinformes de cannabis usando medidas biológicas como variables referentes en diferentes poblaciones (Hjorthøj, Hjorthøj y Nordentoft, 2012). Hemos de considerar dos razones importantes para explicar la ausencia de positivos para cocaína en nuestra muestra: el tamaño muestral pequeño con solo dos positivos para autoinformes y las consideraciones técnicas (vida media corta de los metabolitos -48 a $96 \mathrm{~h}$ - presentes en la muestra de orina) (Moeller, Lee y Kissack, 2008). Una sensibilidad más alta para los autoinformes, comparado con la DRSO, para el uso de benzodiazepinas implica que el uso de benzodiazepinas no se percibe como contraindicado para el trasplante. Además existen limitaciones técnicas para detectar los diferentes compuestos activos en las muestras de orina debido a la pobre reactividad cruzada con los metabolitos conjugados y las benzodiacepinas que no son el diazepam (Melanson, Ptolemy y Wasan, 2013).

Impacto de la DRSO (adherencia/resultados positivos) sobre la supervivencia. Datos anteriores han mostrado que una adherencia baja a los tratamientos y las evaluaciones previas al trasplante hepático es un predictor robusto de alto riesgo de recaída en el consumo de alcohol después del TH (Egawa et al., 2014; Sansone, Bohinc y Wiederman, 2015). En nuestro estudio, encontramos que una adherencia alta a la DRSO durante la valoración para un trasplante hepático estaba correlacionada con la supervivencia del paciente después de ajustar por otro predictores de supervivencia típicos de pacientes con EHA (por ejemplo, MELD). Esta relación también era independiente para el trasplante he- pático entre los pacientes con adherencia alta o negativos persistentes en la DRSO. Además, la presencia de al menos un resultado positivo para alcohol, nicotina u otras sustancias durante el seguimiento no aumentó la mortalidad. El $66 \%$ de los pacientes que habían recibido un trasplante mostró una adherencia alta a DRSO, logrando un resultado positivo en la variable combinada (vivo/trasplante hepático). Por tanto, la valoración de este parámetro dinámico durante el periodo de evaluación de pacientes con EHA en el proceso de trasplante hepático podrá aportar información pronóstica adicional que podría tenerse en cuenta como parte de la valoración de cumplimiento. Se requiere de estudios futuros en este campo para confirmar nuestros datos. De manera sorprendente, el 30,3\% de los pacientes sin adherencia y el $35,1 \%$ de los pacientes que dieron positivo para alcohol en la DRSO fueron receptores de un trasplante hepático. Los pacientes sin adherencia podrían considerarse candidatos válidos a un trasplante porque el equipo priorizó otras características. Un positivo por uso de alcohol no implica que el paciente bebiese durante el periodo entero previo al trasplante, y las recaídas podrían haber sido gestionadas por parte de los profesionales sanitarios durante la evaluación.

Factores asociados con la adherencia a la DRSO. Cuando evaluamos los factores potencialmente asociados con la adherencia a la DRSO durante el seguimiento de los pacientes, encontramos que aquellos afectados por trastornos de personalidad tenían menor adherencia a la DRSO que los pacientes sin esta condición ( $31 \%$ vs. $10 \% ; p=, 02)$. Cuando ajustamos para otras covariables, la presencia de trastornos de personalidad tuvo un impacto negativo en el desarrollo de una adherencia alta a la DRSO en nuestra cohorte ( $\mathrm{RN}, 29 ; \mathrm{p}=, 04)$. Esto no es sorprendente, dado que los pacientes con trastornos de personalidad, en general, tienen un cumplimiento más bajo respecto de su atención sanitaria (Sansone et al., 2015), tratamiento para el trastorno por uso de sustancias (Peles, Schreiber, Domany y Adelson, 2014) o la psicoterapia (Jensen, Mortensen y Lotz, 2014). Es más, es frecuente la comorbilidad de trastornos de personalidad y trastornos de uso de alcohol (Sánchez Autet et al., 2018). Que sepamos, este es el primer estudio que encontró que pacientes con trastornos de la personalidad tienen un cumplimiento más bajo a la detección de sustancias en orina en el proceso de valoración para trasplante hepático. Esto no implica que un trastorno de personalidad sea contraindicado al trasplante, sino que esta población es vulnerable y debería, por tanto, tener un enfoque particular. Otros estudios no han logrado demostrar relación alguna entre los trastornos de personalidad y un pronóstico pobre tras el trasplante hepático en pacientes con EHA (Askgaard et al., 2016; Dom et al., 2015). El apoyo psicológico y farmacológico (Addolorato et al., 2007; Erim et al., 2016) están bajo el foco de los estudios sobre la gestión de los 
candidatos a un trasplante hepático con EHA. No obstante, no existen estudios centrados en la mejora del cumplimiento. La Entrevista Motivacional y la atención centrada en el paciente aumentaron el cumplimiento en otros problemas de salud, como la toma de medicación para la hipertensión o el VIH, los trastornos por uso de alcohol o el seguimiento de pacientes diabéticos. Dicho enfoques quizá merecen ser probados en pacientes con trastornos de personalidad que son candidatos a un trasplante hepático.

Limitaciones y fortalezas. Es relevante reconocer las limitaciones de nuestro estudio. Puede existir un sesgo de memoria, dada su naturaleza retrospectiva. No obstante, en nuestra institución, la valoración de los candidatos a un trasplante hepático sigue un protocolo consolidado, basado en parámetros internacionales y evaluaciones multidisciplinares con reglas estrictas que podrían minimizar el riesgo del sesgo de registro. También supone una limitación la detección de alcohol -en lugar de étil glucurónido- en las muestras de orina, pero esta fue la práctica establecida antes de la generalización del étil glucurónido en nuestro entorno. Los falsos negativos quizás existen a pesar de la variable combinada de referencia y podrían explicar la potencial subestimación del impacto de los resultados positivos en DRSO sobre la supervivencia y el trasplante hepático. Por último, nuestro tamaño muestral pequeño no nos permite ofrecer datos de mayor robustez y potencia estadística. Como mencionamos anteriormente, este estudio fue realizado en un centro único, en el cual, por restricciones geográficas del sistema sanitario local, únicamente se incluyó a aquellos pacientes que podían realizar la prueba de orina de forma regular en nuestra unidad de adicciones. No obstante, nuestro estudio tiene muchas fortalezas. Que sepamos, este es el primer estudio en explorar la precisión añadida al combinar la detección del consumo de alcohol usando ambos autoinformes y la DRSO en candidatos a un trasplante hepático. Analizamos y aportamos información sobre la detección de las drogas usadas con mayor frecuencia en Europa Occidental entre pacientes con EHA evaluados para un trasplante hepático. La adherencia a la DRSO fue baja $(62 \%)$, lo que dificulta aún más interpretar la validez de la DRSO. Mientras que otros estudios se basan en protocolos de investigación, en los cuales la adherencia es estimulada por el Efecto Hawthorne, nuestro estudio tiene un enfoque naturalístico, lo que permite examinar la validez de la DRSO en la práctica en el mundo real. En este sentido, nuestra muestra tenía una prevalencia alta de trastornos psiquiátricos $(30 \%)$, por lo tanto, era más representativo de la práctica real que los estudios prospectivos con criterios de exclusión estrictos o que omitieron datos sobre diagnóstico psiquiátrico. Por último, incluso cuando el impacto del trastorno de personalidad y la adherencia a la DRSO y la supervivencia, respectiva- mente, son resultados preliminares, esta es la primera vez en la cual un estudio muestra esta relación.

\section{Conclusiones}

Ambos autoinformes y DRSO son necesarios para pacientes en valoración para un trasplante hepático. Parece ser que los autoinformes tienen menor sensibilidad, exceptuando para cocaína y benzodiazepinas. Los pacientes con una adherencia buena a la DRSO obtienen mejores resultados, en comparación con los que tienen una adherencia baja. Los pacientes con trastornos de personalidad requieren de más esfuerzos para mejorar su adherencia.

\section{Reconocimientos}

De los autores, el primero (HLP), el segundo (JA), el tercero (EL) y el sexto (AL) han contribuido a la conceptualización, el diseño, la recopilación y la interpretación de datos. Todos los autores contribuyeron al análisis y la interpretación de los datos. Todos los autores han contribuido en la redacción y el contenido intelectual de este documento. Todos los autores han leído y aprobado el artículo para su presentación a la revista.

\section{Financiación}

Dr. Jose Altamirano desea expresar su gratitud al Consejo Nacional de Ciencia y Tecnología (CONACyT, Ciudad México, México) por la financiación parcial de su estancia predoctoral en IDIBAPS.

\section{Conflicto de intereses}

Hugo López-Pelayo ha cobrado honorarios de Lundbeck, Teva y Janssen y subvenciones para viajes de Lundbeck, Otsuka, Lilly, Pfizer, Rovi y Esteve, sin relación alguna con este trabajo. Pablo Barrio ha cobrado honorarios de Lundbeck y Pfizer, y subvenciones para viajes de Pfizer, sin relación alguna con este trabajo. Dr. Antoni Gual ha recibido apoyo financiero de Lundbeck, DyA Pharma y TEVA y cobrado honorarios de Lundbeck, DyA Pharma y Abbivie, sin relación alguna con este estudio. Los demás autores declaran la inexistencia de conflicto de intereses.

\section{Referencias}

Addolorato, G., Leggio, L., Ferrulli, A., Cardone, S., Vonghia, L., Mirijello, A., ... Gasbarrini, G. (2007). Effectiveness and safety of baclofen for maintenance of alcohol abstinence in alcohol-dependent patients with liver cirrhosis: randomised, double-blind controlled study. Lancet, 370, 1915-1922. doi:10.1016/S01406736(07)61814-5. 
Allen, J. P., Wurst, F. M., Thon, N. y Litten, R. Z. (2013). Assessing the drinking status of liver transplant patients with alcoholic liver disease. Liver Transplantation, 19, 369-376. doi:10.1002/lt.23596.

Askgaard, G., Tolstrup, J. S., Gerds, T. A., Hamberg, O., Zierau, L. y Kjær, M. S. (2016). Predictors of heavy drinking after liver transplantation for alcoholic liver disease in Denmark (1990-2013): a nationwide study with competing risks analyses. Scandinavian Journal of Gastroenterology, 51, 225-235. doi:10.3109/00365521.2015.1067903.

Barrio, P., Teixidor, L., Rico, N., Bruguera, P., Ortega, L., Bedini, J. L. y Gual, A. (2016). Urine ethyl glucuronide unraveling the reality of abstinence monitoring in a routine outpatient setting: A cross-sectional comparison with ethanol, self report and clinical judgment. European Addiction Research, 22, 243-248. doi:10.1159/000445741.

Beresford, T. P. y Everson, G. T. (2000). Liver transplantation for alcoholic liver disease: bias, beliefs, 6-month rule, and relapse-but where are the data? Liver Transplantation, 6, 777-778. doi:10.1053/jlts.2000.19027.

Bradley, K. A. y Kivlahan, D. R. (2014). Bringing patient-centered care to patients with alcohol use disorders. JAMA, 311, 1861-1862. doi:10.1001/jama.2014.3629.

Bunzel, B. y Laederach-Hofmann, K. (2000). Solid organ transplantation: are there predictors for posttransplant noncompliance? A literature overview. Transplantation, 70, 711-716.

Burra, P., Senzolo, M., Adam, R., Delvart, V., Karam, V., Germani, G. y Neuberger, J. (2010). Liver transplantation for alcoholic liver disease in Europe: a study from the ELTR (European Liver Transplant Registry). American Journal of Transplantation, 10, 138-148. doi:10.1111/ j.1600-6143.2009.02869.x.

Carbonneau, M., Jensen, L. A., Bain, V. G., Kelly, K., Meeberg, G. y Tandon, P. (2010). Alcohol use while on the liver transplant waiting list: a single-center experience. Liver Transplantation, 16, 91-97. doi:10.1002/1t.21957.

CDER. (2015). Alcoholism: Developing Drugs for Treatment Guidance for Industry. Recuperado de http://www.fda. gov/downloads/drugs/guidancecomplianceregulatoryinformation/guidances/ucm433618.pdf.

Conn, V. S., Ruppar, T. M., Chase, J.-A. D., Enriquez, M. y Cooper, P. S. (2015). Interventions to improve medication adherence in hypertensive patients: systematic review and meta-analysis. Current Hypertension Reports, 17, 94. doi:10.1007/s11906-015-0606-5.

Cuadrado, A., Fábrega, E., Casafont, F. y Pons-Romero, F. (2005). Alcohol recidivism impairs long-term patient survival after orthotopic liver transplantation for alcoholic liver disease. Liver Transplantation, 11, 420-426. doi:10.1002/lt.20386.

Dawwas, M. F., Gimson, A. E., Lewsey, J. D., Copley, L. P. y van der Meulen, J. H. P. (2007). Survival after liver transplantation in the United Kingdom and Ireland compared with the United States. Gut, 56, 1606-1613. doi:10.1136/gut.2006.111369.

de Beaurepaire, R., Lukasiewicz, M., Beauverie, P., Castéra, S., Dagorne, O., Espaze, R., ... Molimard, R. (2007). Comparison of self-reports and biological measures for alcohol, tobacco, and illicit drugs consumption in psychiatric inpatients. European Psychiatry, 22, 540-548. doi:10.1016/j.eurpsy.2007.05.001.

De Gottardi, A., Spahr, L., Gelez, P., Morard, I., Mentha, G., Guillaud, O., ... Dumortier, J. (2007). A simple score for predicting alcohol relapse after liver transplantation: results from 387 patients over 15 years. Archives of Internal Medicine, 167, 1183-1188. doi:10.1001/archinte.167.11.1183.

Di Martino, V., Sheppard, F. y Vanlemmens, C. (2012). Early liver transplantation for severe alcoholic hepatitis. The New England Journal of Medicine, 366, 478-479. doi:10.1056/NEJMc1114241\#SA3.

DiMartini, A., Magill, J., Fitzgerald, M. G., Jain, A., Irish, W., Khera, G. y Yates, W. (2000). Use of a high-risk alcohol relapse scale in evaluating liver transplant candidates. Alcoholism, Clinical and Experimental Research, 24, 1198-1201.

Dom, G., Wojnar, M., Crunelle, C. L., Thon, N., Bobes, J., Preuss, U. W., ... Wurst, F. M. (2015). Assessing and treating alcohol relapse risk in liver transplantation candidates. Alcohol and Alcoholism, 50, 164-172. doi:10.1093/ alcalc/agu096.

EASL clinical practical guidelines: management of alcoholic liver disease. (2012). Journal of Hepatology, 57, 399420. doi:10.1016/j.jhep.2012.04.004.

EASL Clinical Practice Guidelines: Liver transplantation. (2015). Journal of Hepatology, 64, 433-485. doi:10.1016/j. jhep.2015.10.006.

Egawa, H., Nishimura, K., Teramukai, S., Yamamoto, M., Umeshita, K., Furukawa, H. y Uemoto, S. (2014). Risk factors for alcohol relapse after liver transplantation for alcoholic cirrhosis in Japan. Liver Transplantation, 20, 298-310. doi:10.1002/lt.23797.

EMA. (2010). Development of medicinal products for the treatment of alcohol dependence. Recuperado de http://www. ema.europa.eu/docs/en_GB/document_library/ Scientific_guideline/2010/03/WC500074898.pdf

EMCDDA. (2015). European Drug Report 2015: Trends and Developments.

Erim, Y., Böttcher, M., Dahmen, U., Beck, O., Broelsch, C. E. y Helander, A. (2007). Urinary ethyl glucuronide testing detects alcohol consumption in alcoholic liver disease patients awaiting liver transplantation. Liver Transplantation, 13, 757-761. doi:10.1002/lt.21163.

Erim, Y., Böttcher, M., Schieber, K., Lindner, M., Klein, C., Paul, A., ... Helander, A. (2016). Feasibility and acceptability of an alcohol addiction therapy integrated in a transplant center for patients awaiting liver transplan- 
tation. Alcohol and Alcoholism, 51, 40-46. doi:10.1093/ alcalc/agv075.

Gual, A., Contel, M., Segura, L., Ribas, A. y Colom, J. (2001). The ISCA (Systematic Interview of Alcohol Consumption), a new instrument to detect risky drinking. Medicina Clínica, 117, 685-689.

Hill, S. y Kavookjian, J. (2012). Motivational interviewing as a behavioral intervention to increase HAART adherence in patients who are HIV-positive: a systematic review of the literature. AIDS Care, 24, 583-592. doi:10.1080/0 9540121.2011.630354.

Hjorthøj, C. R., Hjorthøj, A. R. y Nordentoft, M. (2012). Validity of Timeline Follow-Back for self-reported use of cannabis and other illicit substances-systematic review and meta-analysis. Addictive Behaviors, 37, 225-233. doi:10.1016/j.addbeh.2011.11.025.

Horrigan, T. J., Piazza, N. J. y Weinstein, L. (1996). The substance abuse subtle screening inventory is more cost effective and has better selectivity than urine toxicology for the detection of substance abuse in pregnancy. Journal of Perinatology, 16, 326-330.

Jensen, H. H., Mortensen, E. L. y Lotz, M. (2014). Drop-out from a psychodynamic group psychotherapy outpatient unit. Nordic Journal of Psychiatry, 68, 594-604. doi:10.310 9/08039488.2014.902499.

Lligoña, A., Freixa, N., Bataller, R., Monràs, M. y Rimola, A. (2009). Clinical guideline for the evaluation of liver transplant candidates with addictions. Gastroenterología y Hepatología, 32, 155-161. doi:10.1016/j.gastrohep.2008.05.003.

Malinchoc, M., Kamath, P. S., Gordon, F. D., Peine, C. J., Rank, J. y ter Borg, P. C. (2000). A model to predict poor survival in patients undergoing transjugular intrahepatic portosystemic shunts. Hepatology, 31, 864-871. doi:10.1053/he.2000.5852.

Martin, P., DiMartini, A., Feng, S., Brown, R. y Fallon, M. (2014). Evaluation for liver transplantation in adults: 2013 practice guideline by the American Association for the Study of Liver Diseases and the American Society of Transplantation. Hepatology, 59, 1144-1165.

Mathurin, P. y Bataller, R. (2015). Trends in the management and burden of alcoholic liver disease. Journal of Hepatology, 62, S38-S46. doi:10.1016/j.jhep.2015.03.006.

Melanson, S. E. F., Ptolemy, A. S. y Wasan, A. D. (2013). Optimizing urine drug testing for monitoring medication compliance in pain management. Pain Medicine, 14, 1813-1820. doi:10.1111/pme.12207.

Moeller, K. E., Lee, K. C. y Kissack, J. C. (2008). Urine drug screening: practical guide for clinicians. Mayo Clinic Proceedings, 83, 66-76. doi:10.4065/83.1.66.

Nanau, R. M., y Neuman, M. G. (2015). Biomolecules and biomarkers used in diagnosis of alcohol drinking and in monitoring therapeutic interventions. Biomolecules, 5, 1339-1385. doi:10.3390/biom5031339.
Niemelä, O. (2016). Biomarker-based approaches for assessing alcohol use disorders. International Journal of Environmental Research and Public Health, 13, 166. doi:10.3390/ijerph13020166.

Oslin, D. W., Pettinati, H. y Volpicelli, J. R. (2002). Alcoholism treatment adherence: older age predicts better adherence and drinking outcomes. The American Journal of Geriatric Psychiatry, 10, 740-747.

Page, T. F., Amofah, S. A., McCann, S., Rivo, J., Varghese, A., James, T., ... Williams, M. L. (2015). Care management medical home center model: Preliminary results of a patient-centered approach to improving care quality for diabetic patients. Health Promotion Practice, 16, 609-616. doi:10.1177/1524839914565021.

Peles, E., Schreiber, S., Domany, Y. y Adelson, M. (2014). Impact of lifetime psychiatric diagnosis on long-term retention and survival of former opiate addicts in methadone maintenance treatment. The World Journal of Biological Psychiatry, 15, 629-635. doi:10.3109/1562297 5.2014.942359.

Piano, S., Marchioro, L., Gola, E., Rosi, S., Morando, F., Cavallin, M., ... Angeli, P. (2014). Assessment of alcohol consumption in liver transplant candidates and recipients: the best combination of the tools available. Liver Transplantation, 20, 815-822. doi:10.1002/lt.23881.

Rehm, J., Gmel, G., Sierra, C. y Gual, A. (2018). Reduction of mortality following better detection of hypertension and alcohol problems in primary health care in Spain. Adicciones, 30, 9-18. doi:10.20882/adicciones.726.

Rustad, J. K., Stern, T. A., Prabhakar, M. y Musselman, D. (2015). Risk factors for alcohol relapse following orthotopic liver transplantation: a systematic review. Psychosomatics, 56, 21-35. doi:10.1016/j.psym.2014.09.006.

Sánchez Autet, M., Garriga, M., Zamora, F. J., González, I., Usall, J., Tolosa, L., ... Arranz, B. (2018). Screening of alcohol use disorders in psychiatric outpatients: influence of gender, age, and psychiatric diagnosis. Adicciones, 30, 251-263. doi:10.20882/adicciones.885.

Sansone, R. A., Bohinc, R. J. y Wiederman, M. W. (2015). Borderline personality symptomatology and compliance with general health care among internal medicine outpatients. International Journal of Psychiatry in Clinical Practice, 19, 132-136. doi:10.3109/13651501.2014.988269.

Staufer, K. y Yegles, M. (2016). Biomarkers for detection of alcohol consumption in liver transplantation. World Journal of Gastroenterology, 22, 3725-3734. doi:10.3748/ wjg.v22.i14.3725.

Stewart, S. H., Koch, D. G., Burgess, D. M., Willner, I. R. y Reuben, A. (2013). Sensitivity and specificity of urinary ethyl glucuronide and ethyl sulfate in liver disease patients. Alcoholism, Clinical and Experimental Research, 37, 150-155. doi:10.1111/j.1530-0277.2012.01855.x.

Surman, O. S., Cosimi, A. B. y DiMartini, A. (2009). Psychiatric care of patients undergoing organ transplan- 
tation. Transplantation, 87, 1753-1761. doi:10.1097/TP. 0b013e3181a754d4.

Telles-Correia, D., Barbosa, A., Mega, I. y Monteiro, E. (2009). Adherence correlates in liver transplant candidates. Transplantation Proceedings, 41, 1731-1734. doi:10.1016/j.transproceed.2009.02.067.

Webzell, I., Ball, D., Bell, J., Sherwood, R. A., Marsh, A., O’Grady, J. G. y Heaton, N. D. (2011). Substance use by liver transplant candidates: an anonymous urinalysis study. Liver Transplantation, 17, 1200-1204. doi:10.1002/ lt. 22370.

Wurst, F. M., Thon, N., Yegles, M., Schrück, A., Preuss, U. W. y Weinmann, W. (2015). Ethanol metabolites: their role in the assessment of alcohol intake. Alcoholism, Clinical and Experimental Research, 39, 2060-2072. doi:10.1111/ acer.12851.

Wurst, F. M., Vogel, R., Jachau, K., Varga, A., Alling, C., Alt, A. y Skipper, G. E. (2003). Ethyl glucuronide discloses recent covert alcohol use not detected by standard testing in forensic psychiatric inpatients. Alcoholism, Clinical and Experimental Research, 27, 471-476. doi:10.1097/01. ALC.0000057942.57330.E2.

\section{Anexo. Abreviaturas}

EHA, enfermedad hepática alcohólica

IC, intervalo de confianza

DSM IV-TR, Manual Diagnóstico y Estadístico de los Trastornos Mentales

TR, tasa de riesgo

HRAR, High-Risk Alcohol Relapse (alto riesgo de recaída al alcoholismo)

MELD, Model for End-stage Liver Disease (modelo para enfermedad hepática terminal)

$\mathrm{RM}$, razón de momios

DRSO, detección regular de sustancias en orina

UBE, unidad de bebida estándar

EGMO, étil glucurónido en muestra de orina 